DSF-12/2003

\title{
BOUNDS FOR THE MASS OF THE HEAVIEST RIGHT-HANDED NEUTRINO IN SO(10) THEORIES
}

\author{
F. Buccella and D. Falcone \\ Dipartimento di Scienze Fisiche, Università di Napoli, Via Cintia, Napoli, Italy
}

\begin{abstract}
By relating the Dirac neutrino mass matrix to the mass of the charged fermions and assuming that the product of the masses of the two lightest left-handed neutrinos is of the order of $\Delta m_{s o l}^{2}$, we derive, within a leptogenesis scenario, a range of values for the mass of the heaviest right-handed neutrino, centered around the scale of $B-L$ symmetry breaking in the $S O(10)$ theory with Pati-Salam intermediate symmetry.
\end{abstract}


Recently the neutrino oscillation [1] solution to the solar neutrino problem has been strongly supported by two experimental results:

a) the rate for the neutral current reaction $\nu+d \rightarrow \nu+p+n$ measured at SNO 2], where the number of active neutrinos is in very good agreement with the flux of $\nu_{e}$ coming from the sun predicted by the standard solar model [3] ;

b) the disappearence of $\bar{\nu}_{e}$ found by KamLAND [4], consistent only with the MSW 5] large mixing solution, which is also the preferred by the combined analysis of the previous experiments [6].

Together with the evidence for the oscillation of atmospheric neutrinos [7] and with the limit by CHOOZ [8] for the disappearence of $\bar{\nu}_{e}$, it brings to a consistent description of the PMNS lepton mixing matrix [1, 9]

$$
U \simeq\left(\begin{array}{ccc}
\frac{\sqrt{3}}{2} & \frac{1}{2} & 0 \\
-\frac{1}{2 \sqrt{2}} & \frac{\sqrt{3}}{2 \sqrt{2}} & \frac{1}{\sqrt{2}} \\
\frac{1}{2 \sqrt{2}} & -\frac{\sqrt{3}}{2 \sqrt{2}} & \frac{1}{\sqrt{2}}
\end{array}\right)
$$

and the square mass differences [10]

$$
\begin{aligned}
\Delta m_{\text {atm }}^{2} & \simeq 2.6 \cdot 10^{-3} \mathrm{eV}^{2}, \\
\Delta m_{\text {sol }}^{2} & \simeq 7 \cdot 10^{-5} \mathrm{eV}^{2} .
\end{aligned}
$$

More severe limitations on the sum of neutrino masses are coming from astrophysical observations 11$]$

$$
\sum\left|m_{i}\right|<0.7 \mathrm{eV}
$$

The smallness of neutrino masses with respect to charged fermions may be understood in the framework of the seesaw mechanism [12], which is a strong indication in favour of $S O(10)$ unification [13], where the existence of $\nu_{R}$ with very large Majorana masses is expected. These particles are also an essential ingredient of the baryogenesis from leptogenesis scenario [14], for which a lower limit of about $5 \cdot 10^{8} \mathrm{GeV}$ for the mass of the lightest $\nu_{R}$ has been found [15]. Indeed, even with a perfect knowledge of the PMNS matrix and of the square mass differences, there are several possibilities for the right handed neutrino mass matrix $M_{R}$, since the Dirac mass matrix $M_{D}$ is unknown, as well as the mass of the lightest $\nu_{L}$ and the relative phases of $m_{i}$.

In $S O(10)$, where the left-handed fermions of each family are classified in a single representation (the spinorial 16), we may try to relate $M_{D}$ to the mass matrices of charged fermions. In the past years we have stressed [16] that in a class of $S O$ (10)-inspired models where one assumes $M_{R 33} \simeq 0$, the largest matrix element of $M_{R}$, namely $M_{R 23}$, takes a 
value of the order of the scale of $B-L$ symmetry breaking found in the unified $S O(10)$ model [17]. Here we want to consider the limits on the value of the mass of the heaviest $\nu_{R}$ which follow from the hypothesis that, at the scale $M_{Z}$,

$$
\operatorname{Det} M_{D}=\frac{\operatorname{Det} M_{e} \cdot \operatorname{Det} M_{u}}{\operatorname{Det} M_{d}},
$$

where $M_{e}, M_{u}$ and $M_{d}$ are the mass matrices for charged leptons and quarks. Eqn.(4) is valid with the Higgs doublet belonging to $\mathbf{1 0}$ representations, but keeps its reliability for matrices like 18

$$
\begin{gathered}
M_{u} \simeq\left(\begin{array}{ccc}
0 & \sqrt{m_{u} m_{c}} & 0 \\
\sqrt{m_{u} m_{c}} & m_{c} & \sqrt{m_{u} m_{t}} \\
0 & \sqrt{m_{u} m_{t}} & m_{t}
\end{array}\right), M_{d} \simeq\left(\begin{array}{ccc}
0 & \sqrt{m_{d} m_{s}} & 0 \\
\sqrt{m_{d} m_{s}} & m_{s} & \sqrt{m_{d} m_{b}} \\
0 & \sqrt{m_{d} m_{b}} & m_{b}
\end{array}\right), \\
M_{D} \simeq \frac{m_{\tau}}{m_{b}}\left(\begin{array}{ccc}
0 & \sqrt{m_{u} m_{c}} & 0 \\
\sqrt{m_{u} m_{c}} & -3 m_{c} & \sqrt{m_{u} m_{t}} \\
0 & \sqrt{m_{u} m_{t}} & m_{t}
\end{array}\right), M_{e} \simeq \frac{m_{\tau}}{m_{b}}\left(\begin{array}{ccc}
0 & \sqrt{m_{d} m_{s}} & 0 \\
\sqrt{m_{d} m_{s}} & -3 m_{s} & \sqrt{m_{d} m_{b}} \\
0 & \sqrt{m_{d} m_{b}} & m_{b}
\end{array}\right),
\end{gathered}
$$

where the factor $m_{\tau} / m_{b}$ comes from the renormalization from the scale of quark-lepton unification to the electroweak scale and the factor -3 in the 2-2 position, typical of Higgs transforming as a $(\mathbf{1 5}, \mathbf{2}, \mathbf{2})$ of $S U(4) \times S U(2) \times S U(2)$, realizes the Georgi-Jarlskog mechanism [19]. For simplicity we omit the $\mathrm{CP}$ violating phase. We assume the normal hierarchy $\left|m_{3}\right| \gg\left|m_{2}\right|,\left|m_{1}\right|$. From Eqn.(4) and the inverse seesaw formula

$$
M_{R} \simeq-M_{D}^{T} M_{L}^{-1} M_{D}
$$

where $M_{L}$ is the effective neutrino mass matrix, we get

$$
\left|\operatorname{Det} M_{R}\right|=\left(\frac{m_{e} m_{\mu} m_{\tau} m_{u} m_{c} m_{t}}{m_{d} m_{s} m_{b}}\right)^{2} \frac{1}{\left|m_{1} m_{2} m_{3}\right|}=1.6 \cdot 10^{30} k \mathrm{GeV}^{3},
$$

where $k$ is the ratio $\Delta m_{\text {sol }}^{2} /\left|m_{1} m_{2}\right|$ and we have considered for the quark masses the values at the $M_{Z}$ scale 20], since the $\operatorname{ratio} \operatorname{Det} M_{u} / \operatorname{Det} M_{d}$ is not modified by renormalization. It should be stressed that Eqn.(4) implies a larger value for Det $M_{D}$ than for $\operatorname{Det} M_{e}$. Moreover, Eqn.(7) implies for the lightest and heaviest right-handed neutrino mass eigenvalues the inequalities

$$
M_{1}<k^{1 / 3} 1.2 \cdot 10^{10}<M_{3}
$$


The first part is consistent with the lower limit found for $M_{1}$ in the baryogenesis from leptogenesis scenario [15],

$$
M_{1}>5 \cdot 10^{8} \mathrm{GeV}
$$

since we have

$$
k>\frac{7 \cdot 10^{-5}}{(2.3)^{2} \cdot 10^{-2}}=1.3 \cdot 10^{-3},
$$

where the upper limit for $\left|m_{1} m_{2}\right|$ comes from Eqn.(3). Then, from Eqns.(8)-(10) we get

$$
1.1 \cdot 10^{9} \mathrm{GeV}<k^{1 / 3} 1.2 \cdot 10^{10}<M_{3}<\frac{k 1.6 \cdot 10^{30}}{25 \cdot 10^{16}}=k 6.4 \cdot 10^{12} \mathrm{GeV}
$$

In principle $k$ could be very large, since $\left|m_{1}\right|$ might be very small, but the fact that

$$
\frac{\Delta m_{\text {sol }}^{2}}{\Delta m_{\text {atm }}^{2}} \simeq 2.7 \cdot 10^{-2}
$$

and the large mixing angles in the PMNS matrix, are in favour of not so different neutrino masses. By taking $k=1$ the geometrical value for the lower and upper limit for $M_{3}$ is $2.8 \cdot 10^{11} \mathrm{GeV}$, very near to the value $2.7 \cdot 10^{11} \mathrm{GeV}$ found for the scale of $B-L$ symmetry breaking in the $S O(10)$ gauge theory with the intermediate $S U(4) \times S U(2) \times S U(2)$ symmetry [17]. The value for the scale of quark-lepton unification, smaller than the mass of the lepto-quarks mediating proton decay, improves the prediction for $m_{b} / m_{\tau}$ [20]. Conversely, by taking that value for $M_{3}$ and the inequality (9) for $M_{1,2}$, one finds

$$
\left|m_{1} m_{2}\right|<\left(3 \cdot 10^{-2}\right)^{2} \mathrm{eV}^{2}
$$

which implies

$$
\sum\left|m_{i}\right|<0.12 \mathrm{eV}
$$

In conclusion, the lower limit on $M_{1}$ in the baryogenesis via leptogenesis scenario and the assumption (7), which is rather reliable in the framework of $S O(10)$, and $k=1$, which is also reliable, since $\sqrt{\Delta m_{\text {sol }}^{2}}$ is less than one order smaller than $\sqrt{\Delta m_{\text {atm }}^{2}}$, estabilish a range for $M_{3}$ centered around the value of the $B-L$ breaking scale in the $S O(10)$ model with Pati-Salam intermediate symmetry 21]. Our conclusion depends also on the assumption (4), which is not necessary true, and from the hypothesis $k \sim 1$, which is reasonable. With smaller $\left|m_{1}\right|$ the lower limit found, which goes as $\left|m_{1}\right|^{-1 / 3}$, has a slow dependance on $\left|m_{1}\right|$, while the upper limit, going as $\left|m_{1}\right|^{-1}$, varies more rapidly. 
Finally, the result we found, which supports the $S O(10)$ theory with Pati-Salam intermediate symmetry, does not exclude SUSY extensions, where lower values of $M_{R}$ than the scale of $B-L$ breaking, which is higher in that case, may be obtained from non-renormalizable couplings [22] $h \phi_{\mathbf{1 6}} \phi_{\mathbf{1 6}} \bar{\psi}_{R}^{c} \psi_{L} / M_{P}+$ h.c., dumped by the Planck scale. 
[1] B. Pontecorvo, Sov. Phys. JETP 6 (1957) 429; 7 (1958) 172

[2] Q.R. Ahmad et al. (SNO Collaboration), Phys. Rev. Lett. 89 (2002) 011301; 011302

[3] J.N. Bahcall and M.H. Pinsonneault, Rev. Mod. Phys. 67 (1995) 781

[4] K. Eguchi et al. (KamLAND Collaboration), Phys. Rev. Lett. 90 (2003) 021802

[5] L. Wolfenstein, Phys. Rev. D 17 (1978) 2369

S.P. Mikheev and A.Yu. Smirnov, Sov. J. Nucl. Phys. 42 (1985) 913

[6] J.N. Bahcall, M.C. Gonzalez-Garcia and C. Pena-Garay, J. High Energy Phys. 02 (2003) 009

[7] Y. Kukuda et al. (SuperKamiokande Collaboration), Phys. Rev. Lett. 81 (1998) 1562

[8] M. Apollonio et al. (CHOOZ Collaboration), Phys. Lett. B 466 (1999) 415

[9] Z. Maki, M. Nakagawa and S. Sakata, Prog. Theor. Phys. 28 (1962) 870

S.M. Bilenky and B. Pontecorvo, Phys. Rep. 41 (1978) 225

[10] G.L. Fogli, Talk at the X International Workshop on Neutrino Telescopes, Venice, March 2003

[11] D.N. Spergel et al., astro-ph/0302209

[12] M. Gell-Mann, P. Ramond and R. Slansky, in Supergravity, eds. P. van Nieuwenhuizen and D. Freedman (North Holland, Amsterdam, 1979)

T. Yanagida, in Proceedings of the Workshop on Unified Theories and Baryon Number in the Universe, eds. O. Sawada and A. Sugamoto (KEK, Tsukuba, 1979)

S.L. Glashow, in Quarks and Leptons, eds. M. Levy et al. (Plenum, New York, 1980)

R.N. Mohapatra and G. Senjanovic, Phys. Rev. Lett. 44 (1980) 912

[13] H. Georgi, in Particles and Fields, ed. C. Carlson (AIP, New York, 1975)

H. Fritzsch and P. Minkowski, Ann. Phys. 93 (1975) 193

[14] M. Fukugita and T. Yanagida, Phys. Lett. B 174 (1986) 45

[15] S. Davidson and A. Ibarra, Phys. Lett. B 535 (2002) 25

W. Buchmuller, P. Di Bari and M. Plumacher, Phys. Lett. B 547 (2002) 128

W. Buchmuller, private communication

[16] M. Abud and F. Buccella, Int. J. Mod. Phys. A 16 (2001) 609

D. Falcone, Phys. Rev. D 66 (2002) 053001 
[17] D. Lee, R.N. Mohapatra, M.K. Parida and M. Rani, Phys. Rev. D 47 (1993) 264

B. Ananthanarayan, K.S. Babu and Q. Shafi, Nucl. Phys. B 428 (1994) 19

F. Acampora, G. Amelino-Camelia, F. Buccella, O. Pisanti, L. Rosa and T. Tuzi, Nuovo Cimento A 108 (1995) 375

F. Buccella and O. Pisanti, hep-ph/9910447

[18] J.L. Chkareuli and C.D. Froggatt, Phys. Lett. B 450 (1999) 158

H. Nishiura, K. Matsuda and T. Fukuyama, Phys. Rev. D 60 (1999) 013006

H. Fritzsch and Z. Xing, Nucl. Phys. B 556 (1999) 49

[19] H. Georgi and C. Jarlskog, Phys. Lett. B 86 (1979) 297

[20] H. Fusaoka and Y. Koide, Phys. Rev. D 57 (1998) 3986

[21] J.C. Pati and A. Salam, Phys. Rev. D 10 (1974) 275

[22] S. Weinberg, Phys. Rev. Lett. 43 (1979) 1566 